\title{
Exogenous thiourea modulates antioxidant defense and glyoxalase systems in lentil genotypes under arsenic stress
}

\author{
Dibyendu Talukdar* \\ Department of Botany, RPM College (University of Calcutta), Uttarpara, Hooghly, West Bengal, India
}

Received: 12.04.2016
Accepted: 05.05.2016
Published: 06.05.2016
*Address for
Correspondence:
Dibyendu Talukdar,
Department of Botany,
RPM College (University
of Calcutta), Uttarpara,
Hooghly, West Bengal, India.
Tel: +9103326630191.
Fax: + 9103326634155.
E-mail: dibyendutalukdar9@
gmail.com

\begin{abstract}
Arsenic (As) is a wide-spread toxic and carcinogenic metalloid, affecting crop productivity worldwide. Lentil, an edible grain legume, is increasingly exposed to soil As contamination. However, our understandings regarding mechanistic details and mitigation strategies against As toxicity in edible legume are extremely poor. The main purpose of this study was to investigate the As-effects and its mitigation by thiourea (TU), a sulfhydryl bioregulator, in lentil. Four widely grown lentil genotypes were grown in nutrient media, supplemented with $30 \mu \mathrm{M}$ sodium As, As $+6.5 \mathrm{mM}$ TU, and As $+13 \mathrm{mM}$ TU, keeping an untreated control for $10 \mathrm{~d}$. As severely affected plant dry weight by accumulating in shoots and roots. However, TU application sequestered As in crop roots and prevented upward translocation of As. TU coordinately modulated glyoxalase (Gly) system I and II (Gly I and II) and ascorbate (AsA)-glutathione (GSH) redox, and antioxidant defense enzymes in both leaves and roots of four genotypes. Elevation of Gly system prevented toxic methylglyoxal overaccumulation, whereas stimulated AsA-GSH cycle enzymes and GSH-s-transferase and catalase effectively scavenged $\mathrm{H}_{2} \mathrm{O}_{2}$ and prevented reactive oxygen species (ROS)-mediated onset of oxidative damage in four genotypes, as was evident from the ROSimaging study. Results suggested exogenous TU stimulated the Gly and antioxidant defense in fine tune against As-induced oxidative damage in lentil genotypes.
\end{abstract}

KEY WORDS: Antioxidant defense, arsenate, glyoxalase system, lentil, thiourea

\section{INTRODUCTION}

Better understanding of mechanistic details of plants' response to environmental toxicants can pave the way to develop safe crop in future. Arsenic (As) is a wide-spread toxic and carcinogenic metalloid. As can induce growth inhibition, low productivity, and poor grain quality by inducing oxidative stress in crop plants (Gunes et al., 2009; Tripathi et al., 2012). Plant experiences oxidative imbalance due to excess generation of reactive oxygen species (ROS) and loss of delicate balance of ROS homeostasis (Finnegan and Chen, 2012). Grain legumes are highly sensitive to As toxicity and being grown in aerobic fields are generally exposed to arsenate (As V) form of As species (Gunes et al., 2009). At the cellular level, As V interferes with normal enzymatic functions and disrupts plant growth and metabolisms (Finnegan and Chen, 2012).

Integration among different defense circuits is pivotal during As-tolerance of crop plants. Ascorbate (AsA) and
GSH are the two key players in non-enzymatic defense components, and their redox states are more important than their total amount in cell (Foyer and Noctor, 2011; Noctor et al., 2012). GSH as a thiol buffer interacts with numerous cellular components and maintains redox homeostasis favorable to reducing environment. The AsA peroxidase (APX), dehydroascorbate (DHA) reductase (DHAR) and glutathione (GSH) reductase (GR) within AsA-GSH cycle and catalases (CAT) and GSH-s-transferase (GST) outside this cycle are the predominant enzymatic antioxidant defense components against ROS-induced oxidative imbalance in cell (Foyer and Noctor, 2003; 2011; Noctor et al., 2012). Besides GSH and GSH-dependent antioxidant defense, methylglyoxal (MG) detoxification systems powered by glyoxalase (Gly) I and II enzymes play significant roles in drought and salinity stress and heavy metal detoxification system in plants (Yadav et al., 2005; Singla-Pareek et al., 2008; Hossain and Fujita, 2010; Hossain et al., 2010). MG is highly toxic to plant cells and by reacting with 
proteins, lipids, carbohydrates, and DNA; they can lead to cell death in the absence of any effective protection. In plants, MG is detoxified mainly via the Gly system that is comprised two enzymes: While Gly I converts MG to S-D-lactoylglutathione (SLG) by utilizing GSH, Gly II converts SLG to D-lactic acid, and during this reaction GSH is regenerated. Overexpressions of the Gly pathway in transgenic tobacco and rice plants experiencing environmental stresses can prevent ROS and MG accumulation by maintaining GSH redox homeostasis and Gly activity levels (Yadav et al., 2005; Singla-Pareek et al., 2008).

Priming of existing defense mechanisms without any genome modification has been found effective during crops' tolerance to stresses (Sahu and Singh 1995; Srivastava et al., 2011). Use of sulfhydryl bio-regulator like thiourea (TU), an ROS-scavenger, has been found highly effective in ameliorating salt, ultraviolet, and heat stress in cereals and oilseeds (Srivastava et al., 2011; Akladious, 2014) and in regulating source-tosink relationship in Indian mustard through alterations in antioxidant defense (Pandey et al., 2013). Among edible legumes, exogenous TU was found to be primarily effective against As-induced oxidative stress in mung bean (Talukdar, 2014), but no such information is available in lentil crop which is extensively grown as grain legume in Indian sub-continent (IIPR, 2011). Primary reports indicate that As exposure can inhibit plant growth and seed yield in lentil genotypes (Talukdar, 2013b; Talukdar and Talukdar, 2014). However, nothing is known regarding mechanistic details of Gly systems and its interaction with GSH-dependent antioxidant defense in any of the grain legume crops under As stress.

Present work was, therefore, designed to: (i) Unravel the response of antioxidant defense and (ii) reveal the modulation of Gly systems under As alone and As + TU applications. The study for the first time indicates coordinated responses between primary antioxidant defense components and MG-detoxification systems during As exposure and their modulations during TUmediated amelioration of As-stress in lentil genotypes. Apart from physiological and biochemical studies, this fact has been confirmed by ROS imaging analysis in this study.

\section{MATERIALS AND METHODS}

\section{Plant Materials and Treatment Protocols}

Fresh and healthy seeds of four lentil genotypes (Lens culinaris Medik. cv. L 9, Pusa 4, K 75, and PL 234) were surface-sterilized with $\mathrm{NaOCl}(0.1 \%$, w/v), washed under running tap water followed by distilled water, and were allowed to germinate in the dark in three separate sets on moistened filter paper at $25^{\circ} \mathrm{C}$. Germinated seedlings were immediately placed in polythene pots (10 plants / pots) containing $300 \mathrm{ml}$ of Hoagland's No. 2 nutrient media following earlier protocol (Talukdar, 2013a), and were allowed to grow for 10 days. The plants were, then, subjected to the following treatment protocols as: (a) Untreated control, (b) $30 \mu \mathrm{M}$ sodium As (As, MW $312.01 \mathrm{~g} / \mathrm{mol}$; technical grade, purity $98.5 \%$, Sigma-Aldrich), (c) $30 \mu \mathrm{M} \mathrm{As}+6.5 \mathrm{mM} \mathrm{TU}$, and (d) $30 \mu \mathrm{M} \mathrm{As}+13 \mathrm{mM}$ TU. Each treatment was replicated four times. TU (Sigma-Aldrich, Bengaluru, India), a sulfhydryl bio-regulator, was used to pre-soak the seeds in the last two protocols. Pilot experiments were carried out to determine the effective concentrations of TU and As. Control and treated plants were allowed to grow for another 10 days. Nutrient solution was refreshed in every alternate day to prevent depletion of nutrients, TU as well as As in the course of the plant's exposure to them. The experiment was carried out in a completely randomized block design in a controlled growth chamber under a $14 \mathrm{~h}$ photoperiod, $26 / 16\left( \pm 2^{\circ} \mathrm{C}\right)$, relative humidity of $72 \pm 2 \%$ and a photosynthesis photon flux density of $180 \mu \mathrm{mol} \mathrm{m}{ }^{-2} \mathrm{~s}^{-1}$. Plants were harvested after a stipulated period. Plant parts were separated and thoroughly washed and oven dried at $72^{\circ} \mathrm{C}$ for $48 \mathrm{~h}$ to measure dry weights of shoots and roots.

\section{Determination of Chlorophyll (Chl) and Total Carotenoids}

Leaf $\mathrm{Chl}$ and carotenoid contents were determined (Lichtenthaler, 1987). Leaf tissue (50 mg) was homogenized in $10 \mathrm{ml}$ chilled acetone (80\%). The homogenate was centrifuged at $4000 \mathrm{~g}$ for $12 \mathrm{~min}$. The absorbance of the supernatant was recorded at 663, 647, and $470 \mathrm{~nm}$ for $\mathrm{Chl} A$, $\mathrm{Chl} \mathrm{B}$, and carotenoids, respectively. The contents were expressed as mg Chl or carotenoids $\mathrm{g}^{-1}$ fresh weight (FW).

\section{Measurement of As Content}

As concentration in dried shoot and root samples was measured by digestion methods $\left(\mathrm{HNO}_{3}-\mathrm{HClO}_{4}\right.$ mixture at 3:1, v/v) using flow injection-hydride generation atomic absorption spectrophotometer (Perkin-Elmer, FIA-HAAS Analyst 400) and keeping Standard Reference Materials of tomato leaves (item number 1573a, from National Institute of Standards and Technology, USA) as part of the quality assurance/ quality control protocol (Talukdar, 2013a). The translocation factor (TF) is the ratio of the level of As in shoots on roots. 


\section{Determination of GSH, Ascorbate Content, and Assay of Antioxidant Defense Enzymes}

Reduced and oxidized form of AsA and GSH were determined according to Law et al. (1983) and Griffith (1980), respectively. For enzyme assay, plant tissue of $250 \mathrm{mg}$ was ground in liquid nitrogen and homogenized in $1 \mathrm{ml}$ of $50 \mathrm{mM} \mathrm{K}$-phosphate buffer (pH 7.8) containing $1 \mathrm{mM}$ ethylene diamine tetraacetic acid (EDTA), $1 \mathrm{mM}$ dithiothreitol (DTT), and 2\% (w/v) polyvinyl pyrrolidone using a chilled mortar and pestle kept in an ice bath. The homogenate was centrifuged at $15,000 \mathrm{~g}$ at $4^{\circ} \mathrm{C}$ for $20 \mathrm{~min}$. Clear supernatant was used for enzyme assays. For measuring APX activity, the tissue was separately grounded in homogenizing medium containing $2.0 \mathrm{mM}$ AsA in addition to the other ingredients. All assays were done at $0-4^{\circ} \mathrm{C}$. Soluble protein content was determined using BSA as a standard (Bradford, 1976).

Superoxide dismutase (SOD) (EC 1.15.1.1) activity was measured by nitro blue tetrazolium (NBT) photochemical assay (Beyer and Fridovich, 1987). One unit of SOD was equal to that amount causing a $50 \%$ decrease of SODinhibited NBT reduction. APX (EC 1.11.1.11) activity ( $\mu \mathrm{mol}$ AsA oxidized $/ \mathrm{min} / \mathrm{mg}$ protein) was assayed following the protocol of Nakano and Asada (1981). Three $\mathrm{ml}$ of the reaction mixture contained $50 \mathrm{mM}$ potassium phosphate buffer ( $\mathrm{pH} 7.0$ ), $0.1 \mathrm{mM}$ EDTA, $0.5 \mathrm{mM}$ AsA, $0.1 \mathrm{mM} \mathrm{H}_{2} \mathrm{O}_{2}$, and $0.1 \mathrm{ml}$ enzyme extract. The $\mathrm{H}_{2} \mathrm{O}_{2}$-dependent oxidation of AsA was followed by a decrease in the absorbance at $290 \mathrm{~nm}(\varepsilon=2.8 / \mathrm{mM} / \mathrm{cm})$. APX activity was expressed as nmol AsA oxidized/min/ mg protein. For DHAR (EC 1.8.5.1) activity assay, the reaction mixture contained $50 \mathrm{mM}$ potassium phosphate buffer (pH 7.0), 0.2 mM DHA, $2.5 \mathrm{mM} \mathrm{GSH}$, and $0.1 \mathrm{mM}$ EDTA in a final volume of $1 \mathrm{ml}$. Reaction was started by addition of suitable aliquots of enzyme extract. The increase in absorbance was recorded at $30 \mathrm{~s}$ intervals for $3 \mathrm{~min}$ at $265 \mathrm{~nm}$. Enzyme activity was expressed as $\mu \mathrm{mol}$ AsA formed $/ \mathrm{min} / \mathrm{mg}$ protein (Nakano and Asada, 1981). For GR (EC 1.6.4.2) assay, enzyme activity was determined by monitoring the GSH-dependent oxidation of nicotinamide adenine dinucleotide phosphate (NADPH) (Carlberg and Mannervik, 1985). In a cuvette, $0.75 \mathrm{ml} 0.2 \mathrm{M}$ potassium phosphate buffer ( $\mathrm{pH} 7.0$ ) containing $75 \mu \mathrm{l}$ NADPH ( $2 \mathrm{mM}), 2 \mathrm{mM}$ EDTA, and $75 \mu \mathrm{l}$ oxidized GSH $(20 \mathrm{mM})$ were mixed. Reaction was initiated by adding $0.1 \mathrm{ml}$ enzyme extract to the cuvette and the decrease in absorbance at $340 \mathrm{~nm}$ was monitored for $2 \mathrm{~min}$. GR specific activity was expressed as nmol NADPH oxidized $/ \mathrm{min} / \mathrm{mg}$ protein. CAT (EC 1.11.1.6) extraction was performed in a $50 \mathrm{mMTris}-\mathrm{HCl}$ buffer. Activity was assayed by measuring the reduction of $\mathrm{H}_{2} \mathrm{O}_{2}$ at $240 \mathrm{~nm}(\varepsilon=39.4 / \mathrm{mM} / \mathrm{cm})$ and $25^{\circ} \mathrm{C}$ as detailed earlier (Talukdar, 2013a; 2013b). For estimation of GSTs (EC 2.5.1.18) specific activity, $1 \mathrm{~g}$ of plant samples was extracted in $5 \mathrm{ml}$ medium containing $50 \mathrm{mM}$ phosphate buffer, $\mathrm{pH}$ 7.5, $1 \mathrm{mM}$ DTT, and $1 \mathrm{mM}$ EDTA. The reaction mixture contained $50 \mathrm{mM}$ phosphate buffer, $\mathrm{pH}$ 7.5, $1 \mathrm{mM}$ 1-chloro-2,4-dinitrobenzene, and the elute equivalent to $100 \mu \mathrm{g}$ of protein. The reaction was initiated with the addition of $1 \mathrm{mM} \mathrm{GSH}$, and formation of S-(2,4-dinitrophenyl) GSH (DNP-GS) was monitored as an increase in absorbance at $334 \mathrm{~nm}$ to calculate the GST specific activity (Li et al., 1995).

Gly I (EC: 4.4.1.5) assay was carried out according to Hossain and Fujita (2010). Briefly, the assay mixture contained $100 \mathrm{mM} \mathrm{K}$-phosphate buffer (pH 7.0), $15 \mathrm{mM}$ magnesium sulfate, $1.7 \mathrm{mM}$ GSH, and $3.5 \mathrm{mM} \mathrm{MG}$ in a final volume of $0.7 \mathrm{ml}$. The reaction was started by the addition of MG, and the activity was calculated at $240 \mathrm{~nm}$ for $1 \mathrm{~min}(\varepsilon=3.37 / \mathrm{mM} / \mathrm{cm})$.

Gly II (EC: 3.1.2.6) activity was determined following Principato et al. (1987) by monitoring the formation of $\mathrm{GSH}$ at $412 \mathrm{~nm}$ for $1 \mathrm{~min}$. The reaction mixture contained 100 mM Tris-HCl buffer (pH 7.2), 0.2 mM DTNB, and $1 \mathrm{mM} \mathrm{SLG}$ in a final volume of $1 \mathrm{ml}$. The reaction was started by the addition of SLG, and the activity was calculated $(\varepsilon=13.6 / \mathrm{mM} / \mathrm{cm})$.

\section{Determination of MG Level}

Plant tissues were homogenized in $5 \%$ perchloric acid and centrifuged at $4^{\circ} \mathrm{C}$ for $10 \mathrm{~min}$ at $11,000 \mathrm{~g}$. The supernatant was decolorized by adding charcoal; then, centrifuged at $11,000 \mathrm{~g}$ for $12 \mathrm{~min}$. The supernatant neutralized by a saturated solution of potassium carbonate at room temperature was used for MG estimation by adding sodium dihydrogen phosphate and $20 \mu \mathrm{l}$ of freshly prepared $0.5 \mathrm{M}$ $\mathrm{N}$-acetyl-L-cysteine to a final volume of $1 \mathrm{ml}$. Formation of the product $N$ - $\alpha$-acetyl-S-(1-hydroxy-2-oxo-prop-1-yl) cysteine was recorded after $10 \mathrm{~min}$ at $288 \mathrm{~nm}$ (Wild et al., 2012). The MG content was calculated by the standard curve and expressed as $\mu \mathrm{mol} / \mathrm{g} / \mathrm{FW}$.

\section{Determination of $\mathrm{H}_{2} \mathrm{O}_{2}$ Content, Membrane Lipid Peroxidation, and Electrolyte Leakage (EL)}

$\mathrm{H}_{2} \mathrm{O}_{2}$ was estimated following Wang et al. (2007) from the absorbance at $410 \mathrm{~nm}$ using a standard curve. Lipid peroxidation rates were determined by measuring the malondialdehyde (MDA) equivalents according to Hodges et al. (1999). EL\% was measured according to DionisioSese and Tobita (1998). 


\section{ROS Imaging}

Detection and imaging of superoxide radicals in leaf and root sections were carried out using the fluorescence probe dihydroethidium (DHE) (Rodr' '1guez-Serrano et al., 2006). Leaf/root segments of approximately $30 \mathrm{~mm}^{2}$ were incubated for $1 \mathrm{~h}$ at $25^{\circ} \mathrm{C}$, in darkness, with $10 \mu \mathrm{M}$ DHE prepared in $5 \mathrm{mMTris}-\mathrm{HCl}$ buffer at $\mathrm{pH} 7.4$, and samples were washed twice with the same buffer for 12 min each. After washing, sections were embedded in a mixture of $15 \%$ acrylamidebisacrylamide stock solution, and $100 \mathrm{~mm}$ thick sections, were cut under $10 \mathrm{mM}$ phosphate-buffered saline (PBS). Sections were then soaked in glycerol: PBS (containing azide) $(1: 1 \mathrm{v} / \mathrm{v})$ and mounted in the same medium for examination with a confocal LASER scanning microscopy (CLSM) system (Carl Zeiss, LSM 780, Bengaluru, India) using standard filters and collection modalities for DHE green fluorescence ( $\lambda$ excitation $488 \mathrm{~nm} ; \lambda$ emission $520 \mathrm{~nm}$ ). $\mathrm{H}_{2} \mathrm{O}_{2}$ was detected by incubation with $25 \mu \mathrm{M}$ 2'7'-dichloro fluorescein diacetate (DCF-DA) (excitation $485 \mathrm{~nm}$, emission $530 \mathrm{~nm}$ ) (Rodr'iguez-Serrano et al., 2006). Preinfused sections with $1 \mathrm{mM}$ tetramethylpiperidinyloxy (TMP), a scavenger of superoxide radicals, and $1 \mathrm{mM}$ AsA, a scavenger of $\mathrm{H}_{2} \mathrm{O}_{2}$ served as negative controls.

\section{Statistical Analysis}

The results are the mean values \pm standard errors of at least four replicates. Multiple comparisons of means were performed by ANOVA (SPSS Inc., version 10), and the means were separated by Duncan's multiple range test with significance level at $P<0.05$. Simple correlation was carried out among different traits using Microsoft Excel data analysis tool pack 2007.

\section{RESULTS}

\section{Changes in Plant Height, Dry Weight, and As Uptake Potential}

Lentil genotypes exhibited significant growth inhibition as both root and shoot length (SL) and dry weights reduced significantly $(P<0.05)$ in comparison to control at $30 \mu \mathrm{M}$ As. Stem and root length (RL) in lentil genotypes were reduced by 2-2.5-fold (Table 1). In L 9 and Pusa 4, while shoot dry weight (SDW) was reduced by about 2-2.7fold root dry weight (RDW) was decreased by about 3-3.3fold (Table 1). SDW in K 75 and PL 234 were declined by nearly 1.8 -fold and 3.1-fold, respectively, whereas RDW was decreased by about 2.5 -fold and 4.5-fold, respectively. Co-application of $6.5 \mathrm{mM}$ TU with As did not change SL and RL as well as SDW and RDW significantly in L 9 and Pusa 4. However, both dry weights increased over control by about 1.5 -fold at As $+13 \mathrm{mMTU}$ in both the genotypes (Table 1). SDW and RDW exhibited upward trend in K 75 and Pl 234 genotypes at As $+6.5 \mathrm{mMTU}$ and further increased at As $+13 \mathrm{mM}$ TU (Table 1). At $30 \mu \mathrm{M}$, As accumulated in marginally higher amount in roots of all four genotypes than that of shoots (shoot/root TF $=1.0$ ). Application of TU at $6.5 \mathrm{mM}$ and $13 \mathrm{mM}$ significantly increased As amount in roots compared to shoots (shoots/ roots TF $<1.0$ ) of all four genotypes but both the $\mathrm{K} 75$ and PL 234 accumulated significantly higher As content than L 9 and Pusa 4 in their roots in presence of TU (Figure 1a).

\section{Changes in Leaf Photosynthetic Pigment}

Leaf $\mathrm{Chl} \mathrm{A}, \mathrm{Chl} \mathrm{A} / \mathrm{B}$ ratio, and carotenoids reduced significantly in four genotypes with different magnitudes

Table 1: Changes in RL (cm/plant), SL (cm/plant), SDW (g/plant), RDW (g/plant) in L 9, Pusa 4, K 75, and PL 234 genotypes of lentils under untreated control, sodium arsenate $(30 \mu \mathrm{M}$ As), As+6.5 mM TU and As+13 mM TU for 10 days treatment duration

\begin{tabular}{|c|c|c|c|c|}
\hline Treatments (traits) & L 9 & Pusa 4 & К 75 & PL 234 \\
\hline Control (RL) & $28.88 \pm 1.8^{\mathrm{bb} \prime}$ & $20.89 \pm 1.3^{\mathrm{cb} \prime}$ & $33.45 \pm 2.1^{\text {aa }}$ & $36.87 \pm 1.9^{\text {aa }}$ \\
\hline As $(R L)$ & $11.56 \pm 1.1^{\mathrm{bb} t}$ & $8.41 \pm 1.0^{\mathrm{bb} \prime}$ & $16.72 \pm 1.3^{\text {aa }}$ & $16.76 \pm 1.3^{\mathrm{aat}}$ \\
\hline As+6.5 mM TU (RL) & $29.03 \pm 1.9^{\mathrm{bb} \prime}$ & $21.03 \pm 1.4^{\mathrm{cb} \prime}$ & $33.57 \pm 2.3^{\text {aa }}$ & $37.00 \pm 2.9^{\mathrm{aa} \prime}$ \\
\hline As+13 mM TU (RL) & $33.03 \pm 2.6^{\text {bat }}$ & $28.08 \pm 1.6^{\mathrm{cb} \prime}$ & $36.51 \pm 2.8^{\text {aa }}$ & $37.88 \pm 3.2^{\mathrm{aat}}$ \\
\hline Control (SL) & $31.45 \pm 1.9^{\mathrm{bb} \prime}$ & $27.56 \pm 1.2^{\mathrm{bb} \prime}$ & $39.11 \pm 2.0^{\text {aa }}$ & $37.22 \pm 1.9^{\text {aat }}$ \\
\hline As $(S L)$ & $12.60 \pm 1.4^{\mathrm{bb} t}$ & $11.02 \pm 1.0^{\mathrm{bb} \prime}$ & $20.08 \pm 1.4^{\text {aa }}$ & $17.01 \pm 1.5^{\text {aa }}$ \\
\hline As+6.5 mM TU (SL) & $31.99 \pm 2.0^{\mathrm{bbr}}$ & $28.11 \pm 1.2^{\mathrm{bb} \prime}$ & $40.12 \pm 2.0^{\text {aa }}$ & $37.76 \pm 1.9^{\mathrm{aat}}$ \\
\hline As+13 mM TU (SL) & $32.11 \pm 2.2^{\mathrm{bb} \prime}$ & $29.13 \pm 1.3^{\mathrm{bb} \prime}$ & $44.32 \pm 2.9^{\text {aa }}$ & $39.52 \pm 2.3^{\mathrm{aa} \prime}$ \\
\hline Control (SDW) & $0.19 \pm 0.04^{\mathrm{bb} \prime}$ & $0.21 \pm 0.04^{\mathrm{bb} \prime}$ & $0.30 \pm 0.06^{\mathrm{aa} \prime}$ & $0.32 \pm 0.08^{\mathrm{aa} \prime}$ \\
\hline As (SDW) & $0.10 \pm 0.02^{\mathrm{bb} \prime}$ & $0.08 \pm 0.01^{\mathrm{bb} \prime}$ & $0.17 \pm 0.03^{\text {aa }}$ & $0.19 \pm 0.02^{\mathrm{aa} \prime}$ \\
\hline As+6.5 mM TU (SDW) & $0.20 \pm 0.04^{\mathrm{bb} \prime}$ & $0.22 \pm 0.04^{\mathrm{bb} \prime}$ & $0.31 \pm 0.07^{\text {aa }}$ & $0.34 \pm 0.09^{\text {aa }}$ \\
\hline As+13 mM TU (SDW) & $0.29 \pm 0.09^{\mathrm{bb} \prime}$ & $0.32 \pm 0.10^{\mathrm{bb} \prime}$ & $0.43 \pm 0.12^{\text {aa }}$ & $0.46 \pm 0.17^{\text {aat }}$ \\
\hline Control (RDW) & $0.24 \pm 0.03^{\mathrm{bb}}$ & $0.26 \pm 0.04^{\mathrm{bb}}$ & $0.36 \pm 0.09^{a a \prime}$ & $0.39 \pm 0.10^{\mathrm{aa}}$ \\
\hline As (RDW) & $0.08 \pm 0.01^{\mathrm{bb} \prime}$ & $0.08 \pm 0.01^{\mathrm{bb} \prime}$ & $0.14 \pm 0.02^{\mathrm{aa} \prime}$ & $0.15 \pm 0.01^{\text {aa }}$ \\
\hline As+6.5 mM TU (RDW) & $0.23 \pm 0.03^{\mathrm{bb}}$ & $0.27 \pm 0.04^{\mathrm{bb}}$ & $0.37 \pm 0.09^{\text {aa }}$ & $0.41 \pm 0.11^{\mathrm{aa}}$ \\
\hline As+13 mM TU (RDW) & $0.36 \pm 0.10^{\mathrm{bb} \prime}$ & $0.39 \pm 0.10^{\mathrm{bb} \prime}$ & $0.47 \pm 0.14^{\text {aa }}$ & $0.50 \pm 0.16^{\mathrm{aa} \prime}$ \\
\hline
\end{tabular}

Means $\pm S E$ of four replicates treatment ${ }^{-1}$. Means followed by different lowercase letters in rows (genotypes) and prime lowercase letters in columns (treatments) are significantly different at $P<0.05$ by ANOVA followed by Duncan's multiple range test. RL: Root length, SL: Shoot length, SDW: Shoot dry weight, RDW: Root dry weight, TU: Thiourea, As: Arsenic 


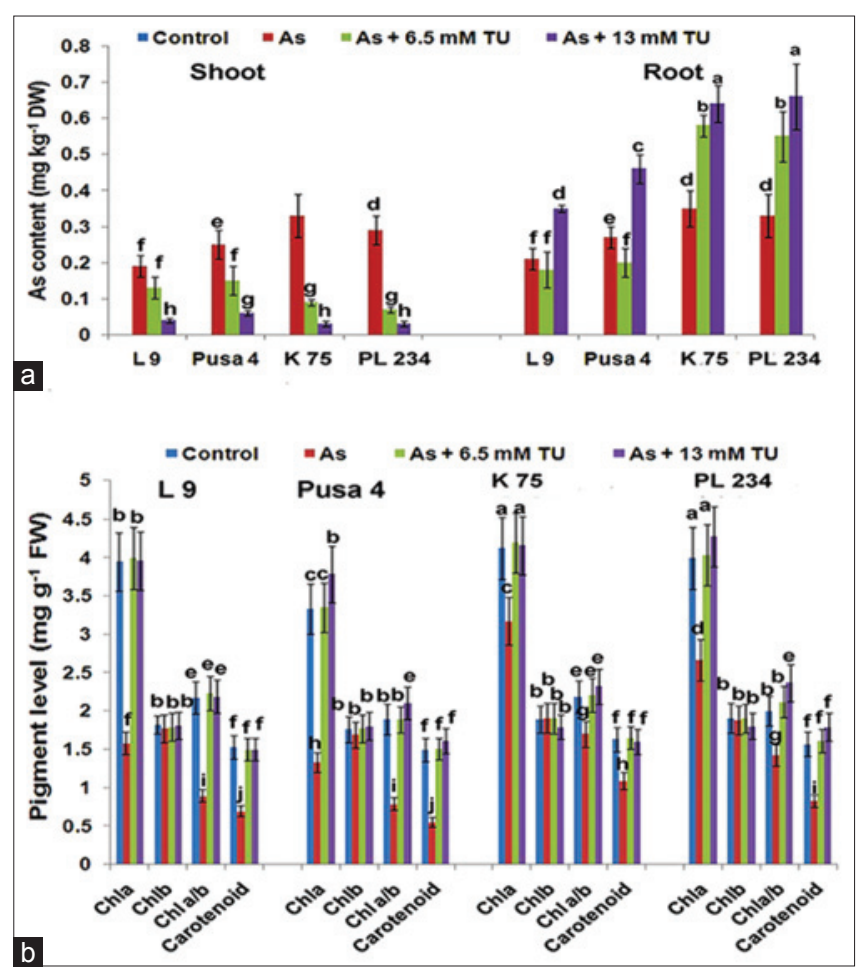

Figure 1: Changes in (a) Arsenic (As) content in shoots and roots and (b) leaf chlorophyll (Chl) A, B, Chl A/B ratio, and carotenoid contents of four lentil genotypes ( $L$ 9, Pusa 4, K 75, and PL 234) under untreated control, $30 \mu \mathrm{M}$ arsenate and As + thiourea (TU) treatments. Data are means \pm standard error of four replicates with different lowercase letters over error bars represent significant differences $(P<0.05)$ at ANOVA followed by Duncan's multiple range test

(Figure 1b). As treatment alone reduced CHL A content by about 2.5-3.2-fold in L 9 and Pusa 4 and 1.3-1.5-fold in K 75 and PL 234 genotypes. CHL B content did not change significantly, but Chl A/B ratio reduced markedly in all genotypes. The carotenoid level was also severely affected with nearly 2.2 (L 9) to 2.7-fold (Pusa 4) decrease and 1.5 (K 75) to 1.9 (PL 234)-fold reduction in lentil genotypes (Table 1). Application of As $+6.5 \mathrm{mMTU}$ and As +13 mMTU considerably restored the photosynthetic pigment levels in four genotypes. Chl A content, Chl A/B ratio, and carotenoid levels even increased significantly in Pusa 4 and PL 234 exposed to As $+13 \mathrm{mM} \mathrm{TU}$ (Figure 1b). Chl A/B ratio was significantly correlated with SDW in L $9(r=0.703, n=12, P<0.05)$, Pusa 4 $(r=0.710, n=12, P<0.05), \mathrm{K} 75(r=0.813, n=12$, $P<0.05)$, and PL $234(r=0.789, n=12, P<0.05)$ genotypes (data not in table).

\section{Response of Antioxidant Defense Components and Gly System}

Foliar and roots AsA and GSH content decreased while DHA and GSH disulfide (GSSG) level increased significantly over control during As exposure alone
(Table 2). Among the four genotypes, As-treatment reduced AsA and GSH levels in both organs of L 9 and Pusa 4 in higher extent than that in K 75 and PL 234 (Table 2). Compared to control, AsA and GSH redox values decreased in all four genotypes, but foliar AsA and GSH redox did not change significantly among the genotypes. Respective redox values in roots, however, were significantly higher in K 75 and PL 234 (Table 2). At As +6.5 mMTU, AsA level was remained low in L 9 and Pusa 4 but comparable to control in K 75 and PL 234. GSH and GSSG content were comparable to control in all four genotypes (Table 2). Upon exposed to As $+13 \mathrm{mMTU}$, root AsA, and GSH content significantly increased, but DHA and GSSG levels decreased in the genotypes. Leaf AsA and GSH did not change significantly in the four genotypes compared to their respective controls. AsA and GSH redox changed, accordingly (Table 2).

Antioxidant enzymatic activities also differed sharply between presence and absence of TU. As exposure alone reduced foliar and root APX, DHAR, GR, and GSTs activities in all four genotypes but the treatment enhanced SOD activity (Figure 2a-e). CAT activity did not change significantly in K 75 and PL 234 but declined significantly in L 9 and Pusa 4 seedlings (Figure 2f). Upon imposition of As $+6.5 \mathrm{mMTU}$, foliar and root activities of APX, DHAR, GR, and GSTs became as per control in all genotypes, but CAT level was remained low. At As + $13 \mathrm{mM} \mathrm{TU}$, activities of five enzymes were significantly higher in roots but were comparable in leaves of all four genotypes (Figure 2a-c, e, f). SOD activity, however, was noticeably low at As $+6.5 \mathrm{mMTU}$, and further reduced at As $+13 \mathrm{mMTU}$ (Figure 2d).

As treatment significantly reduced Gly I and II activities in leaves and roots of L 9 and Pusa 4 genotypes (Figure $3 \mathrm{a}$ and b). Gly I activity did not change significantly, but Gly II level decreased markedly in As-treated K 75 and PL 234 (Figure 3a and b). At As + $6.5 \mathrm{mM} \mathrm{TU}$, Gly I and II activities became comparable to control in leaves of four genotypes but increased markedly in their roots. At As $+13 \mathrm{mM} \mathrm{TU}$, root Gly I and II activity increased significantly in the genotypes, but the foliar activity of both enzymes did not change significantly $(P>0.05)$ in all four genotypes under As +13 mMTU (Figure $3 \mathrm{a}$ and $\mathrm{b}$ ).

\section{Changes in Methylglyoxal, $\mathrm{H}_{2} \mathrm{O}_{2}$, and Oxidative Stress Level}

As treatment alone significantly elevated $\mathrm{MG}$ and other oxidative stress markers such as $\mathrm{H}_{2} \mathrm{O}_{2}$, MDA, and EL\% in leaves and roots of four genotypes (Table 3). MG level 
Table 2: Changes in AsA ( $\mu \mathrm{mol} / \mathrm{g} \mathrm{FW})$, DHA ( $\mu \mathrm{mol} / \mathrm{g} \mathrm{FW})$, glutathione (GSH, $\mathrm{nmol} / \mathrm{g} \mathrm{FW})$, and GSSG (nmol/g FW) content in leaves and roots of L 9, Pusa 4, K 75, and PL 234 genotypes of lentils under untreated control, sodium As (30 $\mu \mathrm{M} \mathrm{As})$, As+6.5 mM (TU) and As+13 mM TU for $10 \mathrm{~d}$ treatment duration

\begin{tabular}{|c|c|c|c|c|c|}
\hline Traits & Treatments & L9 & Pusa 4 & K 75 & PL 234 \\
\hline \multirow[t]{4}{*}{ AsA (leaf) } & Control & $1.09 \pm 0.37^{\text {aa }}$ & $0.98 \pm 0.13^{a b \prime}$ & $1.27 \pm 0.34^{\mathrm{aa} \prime}$ & $1.34 \pm 0.26^{\mathrm{aa} \prime}$ \\
\hline & As & $0.54 \pm 0.07^{\mathrm{bb}}$ & $0.49 \pm 0.06^{\mathrm{bb} \prime}$ & $0.77 \pm 0.07^{\text {ba }}$ & $0.80 \pm 0.08^{\mathrm{ba} \prime}$ \\
\hline & As+6.5 mM TU & $0.58 \pm 0.08^{\mathrm{bb} t}$ & $0.51 \pm 0.07^{\mathrm{bb} \prime}$ & $1.28 \pm 0.38^{\mathrm{aa} \prime}$ & $1.29 \pm 0.18^{\mathrm{aa} \prime}$ \\
\hline & As+13 mM TU & $1.10 \pm 0.38^{\mathrm{ab} \prime}$ & $1.00 \pm 0.15^{\mathrm{ab} \prime}$ & $1.31 \pm 0.39^{\mathrm{aa} \prime}$ & $1.32 \pm 0.28^{\mathrm{aa} \prime}$ \\
\hline \multirow[t]{4}{*}{ AsA (root) } & Control & $0.99 \pm 0.28^{\text {bat }}$ & $0.97 \pm 0.16^{\text {ba }}$ & $1.03 \pm 0.19^{\text {ba }}$ & $1.05 \pm 0.21^{\mathrm{ba} \prime}$ \\
\hline & As & $0.50 \pm 0.06^{\mathrm{cb} \prime}$ & $0.51 \pm 0.05^{\mathrm{cb} \prime}$ & $0.71 \pm 0.11^{\mathrm{ca} \prime}$ & $0.69 \pm 0.10^{\mathrm{ca} \prime}$ \\
\hline & As+6.5 mM TU & $1.01 \pm 0.29^{\text {ba }}$ & $0.97 \pm 0.18^{\text {ba }}$ & $1.05 \pm 0.31^{\mathrm{ba} \prime}$ & $1.07 \pm 0.34^{\mathrm{ba} \prime}$ \\
\hline & As+13 mM TU & $1.87 \pm 0.33^{\mathrm{ab}}$ & $1.85 \pm 0.34^{\mathrm{ab} \prime}$ & $1.99 \pm 0.45^{\mathrm{aa} \prime}$ & $1.98 \pm 0.39^{\mathrm{aa} \prime}$ \\
\hline \multirow[t]{4}{*}{ DHA (leaf) } & Control & $0.12 \pm 0.03^{\text {ba }}$ & $0.14 \pm 0.02^{\mathrm{ba} \prime}$ & $0.14 \pm 0.02^{\mathrm{ba} \prime}$ & $0.15 \pm 0.03^{\text {ba }}$ \\
\hline & As & $0.39 \pm 0.05^{\mathrm{ac}}$ & $0.60 \pm 0.06^{\mathrm{aa} \prime}$ & $0.45 \pm 0.07^{\mathrm{ac}}$ & $0.53 \pm 0.09^{\mathrm{ab}}$ \\
\hline & As+6.5 mM TU & $0.17 \pm 0.02^{\mathrm{ab} \prime}$ & $0.17 \pm 0.07^{a b \prime}$ & $0.22 \pm 0.07^{\text {aa } \prime}$ & $0.27 \pm 0.08^{\text {aa }}$ \\
\hline & As+13 mM TU & $0.09 \pm 0.02^{\text {ba }}$ & $0.11 \pm 0.04^{\text {ca }}$ & $0.10 \pm 0.03^{\mathrm{ca} \prime}$ & $0.13 \pm 0.02^{\mathrm{bat}}$ \\
\hline \multirow[t]{4}{*}{ DHA (root) } & Control & $0.11 \pm 0.02^{\text {ba }}$ & $0.11 \pm 0.03^{\text {ca }}$ & $0.12 \pm 0.02^{\text {ba }}$ & $0.12 \pm 0.04^{\text {bat }}$ \\
\hline & As & $0.60 \pm 0.08^{a a \prime}$ & $0.68 \pm 0.09^{a a}$ & $0.48 \pm 0.03^{a b \prime}$ & $0.47 \pm 0.03^{\mathrm{ab} \prime}$ \\
\hline & As+6.5 mM TU & $0.16 \pm 0.06^{\mathrm{aa}}$ & $0.15 \pm 0.05^{\text {ba }}$ & $0.13 \pm 0.03^{\text {ba }}$ & $0.15 \pm 0.05^{\mathrm{aa} \prime}$ \\
\hline & As+13 mM TU & $0.10 \pm 0.02^{\mathrm{ba}}$ & $0.09 \pm 0.01^{\mathrm{ca}}$ & $0.10 \pm 0.02^{\mathrm{ba} \mathrm{a}^{\prime}}$ & $0.11 \pm 0.01^{\mathrm{ba}}$ \\
\hline \multirow[t]{4}{*}{ AsA redox (leaf) } & Control & $0.901 \pm 0.04^{\text {ba }}$ & $0.876 \pm 0.05^{\mathrm{bb} \prime}$ & $0.900 \pm 0.05^{\text {aa }}$ & $0.899 \pm 0.05^{\mathrm{aa} \prime}$ \\
\hline & As & $0.580 \pm 0.06^{\mathrm{db} t}$ & $0.450 \pm 0.02^{c c \prime}$ & $0.670 \pm 0.03^{\text {ca }}$ & $0.601 \pm 0.02^{\mathrm{cb} t}$ \\
\hline & As+6.5 mM TU & $0.773 \pm 0.06^{\mathrm{cb} \prime}$ & $0.750 \pm 0.04^{\mathrm{cb} \prime}$ & $0.853 \pm 0.09^{\mathrm{ba}}$ & $0.830 \pm 0.08^{\text {bat }}$ \\
\hline & As+13 mM TU & $0.924 \pm 0.09^{\mathrm{aa}}$ & $0.900 \pm 0.08^{\mathrm{aa} \prime}$ & $0.930 \pm 0.09^{\mathrm{aa}}$ & $0.910 \pm 0.09^{\mathrm{aa}}$ \\
\hline \multirow[t]{4}{*}{ AsA redox (root) } & Control & $0.900 \pm 0.05^{\mathrm{aa} \prime}$ & $0.899 \pm 0.05^{\mathrm{aa} \prime}$ & $0.896 \pm 0.05^{\mathrm{ba}}$ & $0.897 \pm 0.04^{\mathrm{ba} \prime}$ \\
\hline & As & $0.455 \pm 0.05^{\mathrm{cb} \prime}$ & $0.429 \pm 0.07^{\mathrm{cb} \prime}$ & $0.597 \pm 0.08^{\mathrm{ca} \prime}$ & $0.595 \pm 0.08^{\text {ca }}$ \\
\hline & As+6.5 mM TU & $0.863 \pm 0.08^{\text {ba }}$ & $0.867 \pm 0.08^{\text {ba }}$ & $0.890 \pm 0.09^{\mathrm{ba}}$ & $0.877 \pm 0.08^{\mathrm{ba} \prime}$ \\
\hline & As+13 mM TU & $0.950 \pm 0.09^{\mathrm{aa}}$ & $0.954 \pm 0.09^{\mathrm{aa} \prime}$ & $0.952 \pm 0.09^{\mathrm{aa}}$ & $0.947 \pm 0.09^{\mathrm{aa}}$ \\
\hline \multirow[t]{4}{*}{ GSH (leaf) } & Control & $177.8 \pm 3.9^{\mathrm{aa} \prime}$ & $181.9 \pm 4.0^{\mathrm{aa} \prime}$ & $209.6 \pm 5.2^{\mathrm{aa} \prime}$ & $212.8 \pm 5.8^{\mathrm{aa} \prime}$ \\
\hline & As & $88.3 \pm 2.5^{\mathrm{bb} \prime}$ & $89.5 \pm 2.6^{\mathrm{bb} \prime}$ & $100.3 \pm 3.8^{\mathrm{ba} \prime}$ & $105.2 \pm 3.2^{\mathrm{ba} \prime}$ \\
\hline & As+6.5 mM TU & $175.5 \pm 4.1^{a b^{\prime}}$ & $179.8 \pm 4.0^{a b^{\prime}}$ & $210.2 \pm 5.5^{\text {aa }}$ & $209.7 \pm 5.3^{\text {aa }}$ \\
\hline & As+13 mM TU & $170.4 \pm 3.9^{\mathrm{ab} \prime}$ & $180.4 \pm 3.7^{a b \prime}$ & $205.9 \pm 4.7^{\text {aa }}$ & $210.2 \pm 5.2^{\mathrm{aa} \prime}$ \\
\hline \multirow[t]{4}{*}{ GSSG (leaf) } & Control & $19.8 \pm 1.8^{\mathrm{ba} \prime}$ & $22.7 \pm 1.6^{\text {ba }}$ & $25.3 \pm 2.3^{\mathrm{ba} \prime}$ & $20.9 \pm 1.9^{\text {ba }}$ \\
\hline & As & $59.9 \pm 2.6^{\text {aa }}$ & $61.8 \pm 3.1^{\mathrm{aa} \prime}$ & $67.7 \pm 2.8^{\mathrm{aa} \prime}$ & $60.3 \pm 2.2^{\text {aa } \prime}$ \\
\hline & As $+6.5 \mathrm{mM}$ TU & $20.1 \pm 1.6^{\text {ba }}$ & $22.5 \pm 1.5^{\text {ba }}$ & $25.6 \pm 2.2^{\text {ba }}$ & $21.0 \pm 2.0^{\text {ba }}$ \\
\hline & As+13 mM TU & $19.7 \pm 1.8^{\text {ba }}$ & $20.8 \pm 1.8^{\mathrm{ba} \prime}$ & $24.8 \pm 2.2^{\text {bat }}$ & $18.8 \pm 1.8^{\mathrm{ca} \prime}$ \\
\hline \multirow[t]{4}{*}{ GSH (root) } & Control & $109.5 \pm 1.5^{\mathrm{bc}}$ & $170.8 \pm 1.6^{\mathrm{bb}}$ & $198.5 \pm 1.9^{\text {ba }}$ & $189.8 \pm 1.8^{\mathrm{ba} \prime}$ \\
\hline & As & $70.8 \pm 2.1^{\mathrm{cb} \prime}$ & $70.3 \pm 2.3^{\mathrm{cb} \prime}$ & $106.8 \pm 1.5^{\mathrm{ca} \prime}$ & $103.5 \pm 1.3^{\mathrm{ca} \prime}$ \\
\hline & As+6.5 mM TU & $110.0 \pm 1.5^{\mathrm{bc}}$ & $171.1 \pm 1.7^{\mathrm{bb} \prime}$ & $200.1 \pm 2.2^{\mathrm{ba} \prime}$ & $190.3 \pm 1.9^{\text {ba }}$ \\
\hline & As+13 mM TU & $177.7 \pm 2.4^{a}$ & $190.8 \pm 2.6^{a}$ & $223.7 \pm 3.1^{\mathrm{a}}$ & $228.9 \pm 3.1^{a}$ \\
\hline \multirow[t]{4}{*}{ GSSG (root) } & Control & $17.6 \pm 1.1^{\mathrm{ba} \prime}$ & $20.7 \pm 1.5^{\text {ba }}$ & $18.6 \pm 1.4^{\text {bat }}$ & $22.6 \pm 2.4^{\text {ba }}$ \\
\hline & As & $79.8 \pm 2.3^{\text {aa } \prime}$ & $72.3 \pm 2.5^{\mathrm{aa} \prime}$ & $78.7 \pm 2.2^{\text {aa }}$ & $84.4 \pm 3.8^{\mathrm{aa} \prime}$ \\
\hline & As+6.5 mM TU & $17.5 \pm 1.1^{\mathrm{ba} \prime}$ & $21.0 \pm 2.1^{\mathrm{ba} \prime}$ & $18.5 \pm 1.3^{\mathrm{ba} \prime}$ & $23.1 \pm 2.3^{\text {ba }}$ \\
\hline & As+13 mM TU & $8.78 \pm 0.12^{\text {ca' }}$ & $9.11 \pm 0.15^{\mathrm{ca} \prime}$ & $10.0 \pm 0.10^{\text {ca }}$ & $6.18 \pm 0.67^{\mathrm{cb} \prime}$ \\
\hline \multirow[t]{4}{*}{ GSH redox (leaf) } & Control & $0.899 \pm 0.09^{\text {aa }}$ & $0.889 \pm 0.09^{\text {aa }}$ & $0.892 \pm 0.09^{\mathrm{aa} \prime}$ & $0.910 \pm 0.09^{\mathrm{aa} \prime}$ \\
\hline & As & $0.594 \pm 0.05^{\text {ba }}$ & $0.591 \pm 0.06^{\text {ba }}$ & $0.595 \pm 0.06^{\text {ba }}$ & $0.633 \pm 0.06^{\text {ba }}$ \\
\hline & As+6.5 mM TU & $0.897 \pm 0.08^{\mathrm{aa} \prime}$ & $0.891 \pm 0.09^{\text {aa }}$ & $0.891 \pm 0.09^{\text {aa }}$ & $0.909 \pm 0.09^{\text {aa }}$ \\
\hline & As+13 mM TU & $0.896 \pm 0.08^{\mathrm{aa} \prime}$ & $0.900 \pm 0.09^{\text {aa }}$ & $0.892 \pm 0.08^{\mathrm{aa} \prime}$ & $0.917 \pm 0.09^{a a}$ \\
\hline \multirow[t]{4}{*}{ GSH redox (root) } & Control & $0.861 \pm 0.08^{\text {ba }}$ & $0.891 \pm 0.08^{\text {bal }}$ & $0.914 \pm 0.09^{\text {ba }}$ & $0.893 \pm 0.08^{\mathrm{aa} \prime}$ \\
\hline & As & $0.470 \pm 0.05^{\mathrm{cb} \prime}$ & $0.493 \pm 0.05^{\mathrm{cb} \prime}$ & $0.575 \pm 0.06^{\text {cat }}$ & $0.551 \pm 0.06^{\mathrm{ba}}$ \\
\hline & As+6.5 mM TU & $0.863 \pm 0.08^{\mathrm{bb} \prime}$ & $0.891 \pm 0.09^{\text {ba }}$ & $0.915 \pm 0.09^{\text {ba }}$ & $0.892 \pm 0.08^{\mathrm{aa} \prime}$ \\
\hline & As+13 mM TU & $0.953 \pm 0.09^{\mathrm{aa} \prime}$ & $0.954 \pm 0.09^{\text {aa }}$ & $0.957 \pm 0.09^{\text {aa }}$ & $0.974 \pm 0.09^{a a}$ \\
\hline
\end{tabular}

Means $\pm S E$ of four replicates treatment ${ }^{-1}$. Means followed by different lowercase letters in columns and lower case letters with prime in rows are significantly different at $P<0.05$ by ANOVA following Duncan's multiple range test. AsA: Ascorbate, DHA: Dehydroascorbate, GSSG: Glutathione disulfide, SE: Standard error, FW: Fresh weight, As: Arsenic, SE: Standard error

reduced significantly in both organs of all four genotypes on imposition of As $+6.5 \mathrm{mM}$ TU and further reduced at As +13 mMTU (Table 3). Foliar and root $\mathrm{H}_{2} \mathrm{O}_{2}$, MDA and EL\% increased to the tune of 3-6-fold in leaves and roots of the genotypes exposed to As only. During As + TU treatment, $\mathrm{H}_{2} \mathrm{O}_{2}$ level decreased substantially which was accompanied by normal (comparable to control) to reduced level of MDA and EL\% in leaves and roots of all four genotypes (Table 3).

\section{ROS Imaging Study}

In leaves and roots of untreated controls, faint red (superoxide) and green $\left(\mathrm{H}_{2} \mathrm{O}_{2}\right)$ fluorescence was detected in the vascular region (Figure 4a, b, f, g). However, in As-treated genotypes, bright red and green fluorescence was detected in vascular bundle regions, endodermis, sclerenchyma, mesophyll (leaf), and epidermis (Figure 4c-e, h, i). Preincubation of sections with $1 \mathrm{mM}$ TMP (a superoxide 


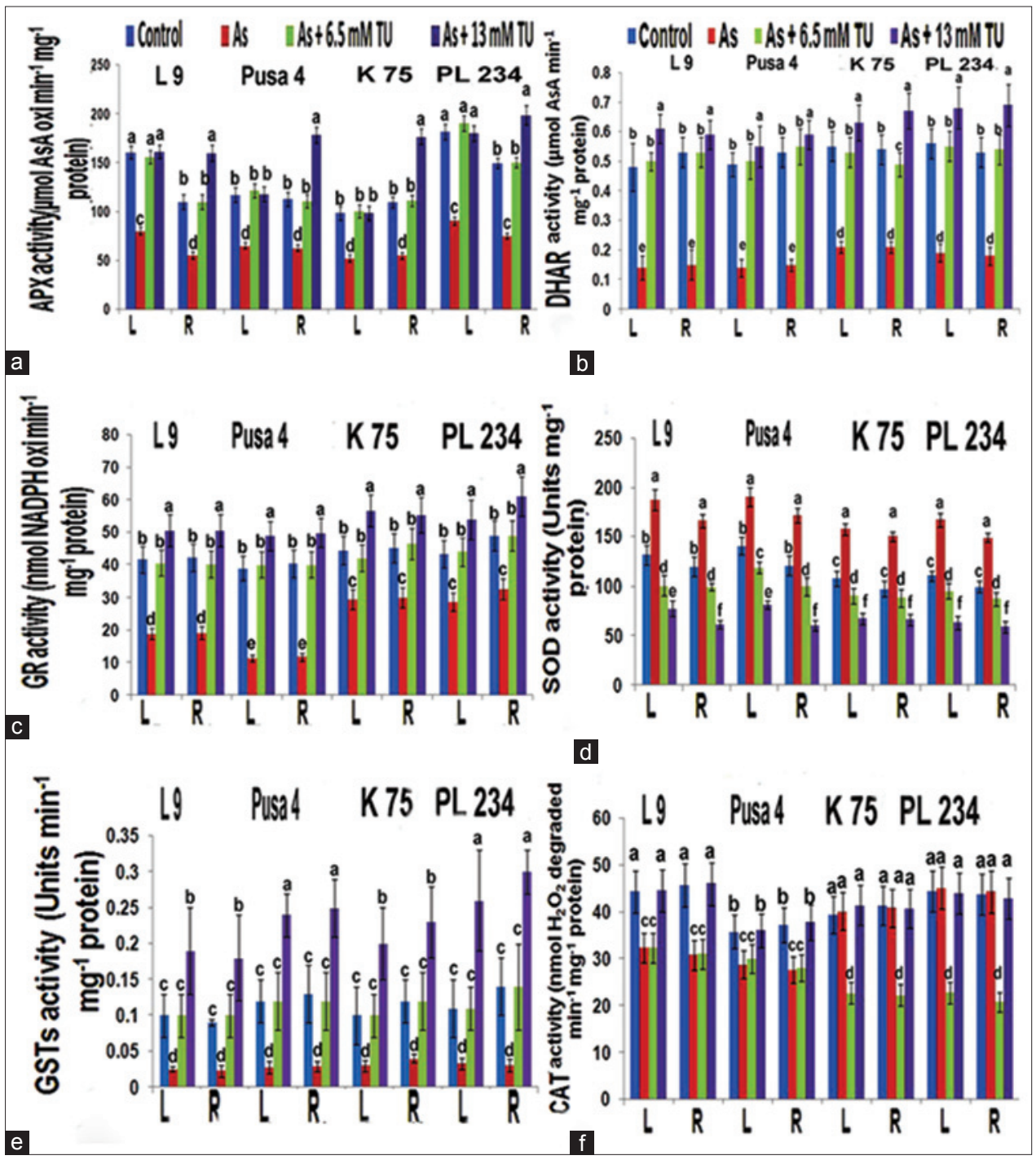

Figure 2: Changes in (a) Ascorbate peroxidase, (b) dehydroascorbate reductase, (c) glutathione (GSH) reductase, (d) superoxide dismutase, (e) GSH-s-transferases, and (f) catalases activities in leaves and roots of four lentil (L 9, Pusa 4, K 75, and PL 234) genotypes under untreated control, $30 \mu \mathrm{M}$ arsenate and arsenic + thiourea treatments. Data are means \pm standard error of four replicates with different lowercase letters over error bars represent significant differences $(P<0.05)$ at ANOVA followed by Duncan's multiple range test

scavenger) and AsA (an $\mathrm{H}_{2} \mathrm{O}_{2}$ scavenger) resulted in significant reduction of the fluorescence (Figure 4j-1). Upon As +6.5 mM TU, mild DHE (red) and DCF-DA (green) fluorescence was localized only in vascular bundle and epidermis of four genotypes which was further reduced at $13 \mathrm{mMTU}+$ As (Figure 5a-h).

\section{DISCUSSION}

Growth inhibition is one of the significant primary physiological phenomena during As-toxicity of crop plants (Stoeva et al., 2005; Gupta et al., 2008; Malik et al., 2012; Talukdar, 2013b). In this study, reduction in dry weight was mainly due to decrease in stem and RL in four genotypes exposed to As-treatment alone. However, genotypes differed significantly in their responses toward
As-treatment. The L 9 and Pusa 4 suffered greater inhibition of SL and RL as well as SDW and RDW than K 75 and PL 234. The present finding was consistent with earlier reports on As-induced growth inhibition of lentil, grass pea, common beans, chickpea, rice, and mustard crops with significant genotypic differences (Chaturvedi, 2006; Gunes et al., 2009; Talukdar, 2013c; 2015; Talukdar and Talukdar, 2014). Here, upon application of TU, significant improvement of plant growth was evident from comparable SDW and RDW at $6.5 \mathrm{mM}$, and thereafter, their increase over control in L 9 and Pusa 4 at $13 \mathrm{mM}$. More significantly, in the case of both $\mathrm{K}$ 75 and PL 234, SDW and RDW increased over control at $6.5 \mathrm{mM}$ TU and further increased at $13 \mathrm{mM}$ TU. Interestingly, As accumulation pattern in the absence of TU was nearly comparable between roots and shoots 


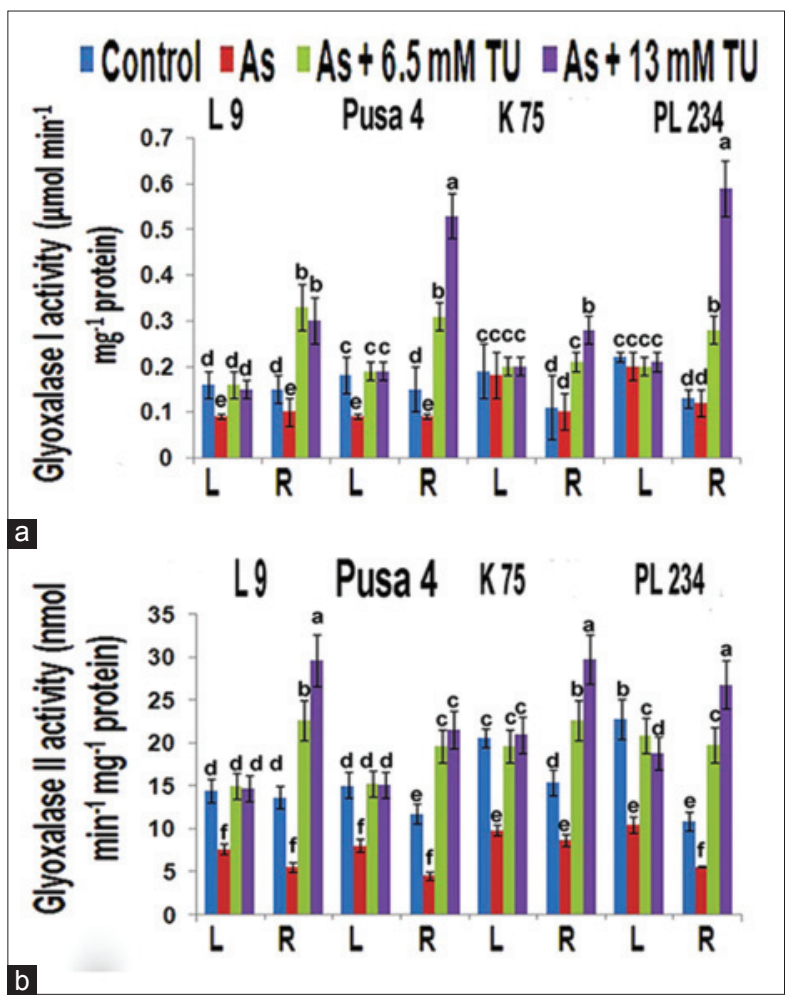

Figure 3: Changes in (a) Glyoxalase (Gly) I and (b) Gly II activities in leaves and roots of two lentils (L 9 and Pusa 4) and grass pea (BioL 202 and Prateek) genotypes at untreated control, $30 \mu \mathrm{M}$ arsenate, and arsenic + thiourea treatment. Data are means \pm standard error of four replicates with different lowercase letters over error bars represent significant differences $(P<0.05)$ at ANOVA followed by Duncan's multiple range test

$(\mathrm{TF}=1)$ in all four genotypes but roots accumulated far greater amount than shoots (shoots/roots TF $<1.0$ ) in the presence of TU. The results suggested that TU application facilitated greater sequestration of As in crop roots and prevented upward translocation of As into photosynthetic parts. Comparing L 9 and Pusa 4, K 75, and PL 234 genotypes performed better, exhibiting higher growth traits but accumulating greater amount of As in their roots. The results pointed out genotypic differences for As accumulation pattern in present lentil crops.

As-induced growth inhibition may be related to severe reduction in photosynthetic apparatus as $\mathrm{Chl} \mathrm{A} / \mathrm{B}$ ratio, one of the significant biomarkers of plant photosynthetic ability, decreased due to reduction in Chl A level, and is significantly correlated with SDW in four genotypes. The reduction in carotenoids level suggested As-induced decline and/or damage in photosynthetic and antioxidant ability of the genotypes. The decrease in total Chl content is one of the early symptoms of metal (loid)-induced toxicity in crop plants (Azizur Rahman et al., 2007; Talukdar, 2013b). Injuries in the photosynthetic apparatus

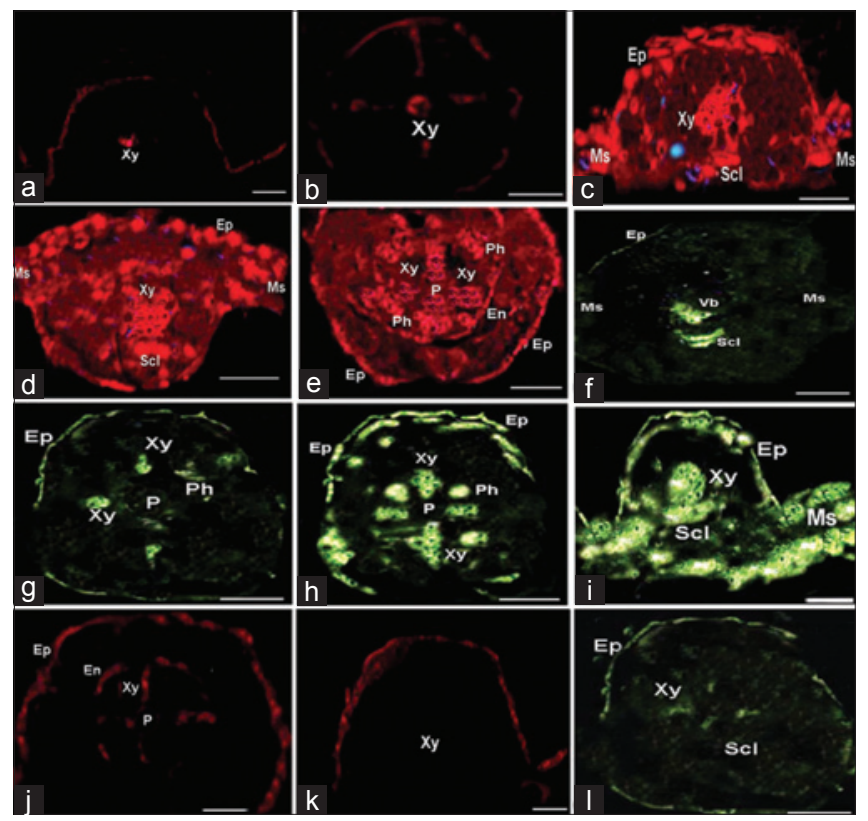

Figure 4: Representative photographs of reactive oxygen species imaging study by Confocal LASER Scanning Microscopy with dihydroethidium (superoxide radicals-specific) and 2'7'-dichloro fluorescein diacetate $\left(\mathrm{H}_{2} \mathrm{O}_{2}\right.$-specific) dye showing mild red fluorescence in sections of (a) Control leaf, (b) control roots, bright red fluorescence in $30 \mu \mathrm{M}$ arsenate (As)-treated (c,d) leaf sections (L 9 and $K$ 75), (e) root sections of Pusa 4, mild green fluorescence in control (f) leaf and ( $g$ ) root sections, bright green in As-treated (h) root, (i) leaf sections, reduced red fluorescence in (j) roots, (k) leaf and green fluorescence in (I) roots of Pusa 4 genotype after tetramethylpiperidinyloxy and ascorbate treatments (negative control). Blue region in Figure (c-e) indicates chlorophyll autofluorescence (chlorophyll A and $B, \lambda$ excitation 429 and $450 \mathrm{~nm} ; \lambda$ emission 650 and $670 \mathrm{~nm}$ ). The results are representative of at least four independent experiments. Ep: Epidermis; En: endodermis, Ms: mesophyll cells; Scl: sclerenchyma; Xy: xylem; Ph-phloem; P-pith. Bars $=50 \mu \mathrm{m}$

result in many negative consequences such as reduction in plant growth, yield, and impediment in plant survival (Azizur Rahman et al., 2007; Singh et al., 2007; Ashraf and Harris, 2013). The improvement of plant growth by elevated dry mass accumulation and the sequestration of lion's share of As into roots by present genotypes after TU application was in fine tune with improved $\mathrm{Chl} \mathrm{A,} \mathrm{Chl}$ $\mathrm{A} / \mathrm{B}$ ratio, and carotenoid content at As + TU treatment protocol. As-induced reduction in $\mathrm{Chl} \mathrm{A}$ and $\mathrm{Chl} \mathrm{A} / \mathrm{B}$ ratio was observed in As-exposed edible legumes, cereals, and other crops but unlike the present circumstances in most of these cases carotenoid levels were not affected by As exposure (Guo et al., 2005; Talukdar and Talukdar, 2013; 2014). The reasons for reduced photosynthetic pigment might be due to changes in chloroplast and carotenoid structure and/or inhibited biosynthesis of $\mathrm{Chl} /$ carotenoid or its precursors (Azizur Rahman et al., 2007). Present results, thus, suggested strong ameliorative capability of TU in protection of photosynthetic pigment content which obviously had a positive effect on plant 
Table 3: Changes in MG ( $\mu \mathrm{mol} / \mathrm{g} \mathrm{FW}), \mathrm{H}_{2} \mathrm{O}_{2}(\mu \mathrm{mol} / \mathrm{g} \mathrm{FW}), \mathrm{MDA}$ (nmol/g FW), and EL\% in leaves and roots of L 9, Pusa 4, $\mathrm{K} 75$, and PL 234 genotypes of lentils under untreated control, sodium arsenate $(30 \mu \mathrm{M} \mathrm{As})$, As+6.5 mM TU and As+13 mM TU for 10 days treatment duration

\begin{tabular}{|c|c|c|c|c|c|c|}
\hline Traits & Treatments & Organ & L 9 & Pusa 4 & BioL 202 & Prateek \\
\hline \multirow[t]{8}{*}{$M G$} & \multirow[t]{2}{*}{ Control } & Leaves & $18.9 \pm 1.8^{b}$ & $20.4 \pm 2.0^{b}$ & $31.8 \pm 3.2^{\mathrm{a}}$ & $33.6 \pm 3.7^{a}$ \\
\hline & & Roots & $13.7 \pm 1.4^{\mathrm{b}}$ & $15.5 \pm 1.6^{b}$ & $19.7 \pm 1.9^{\mathrm{a}}$ & $21.4 \pm 2.1^{a}$ \\
\hline & \multirow[t]{2}{*}{ As } & Leaves & $23.6 \pm 2.3^{b}$ & $39.8 \pm 4.1^{a}$ & $40.7 \pm 4.0^{a}$ & $39.7 \pm 4.1^{a}$ \\
\hline & & Roots & $29.8 \pm 2.7^{b}$ & $43.2 \pm 4.3^{a}$ & $40.9 \pm 4.1^{\mathrm{a}}$ & $45.8 \pm 4.6^{a}$ \\
\hline & \multirow[t]{2}{*}{ As+6.5 mM TU } & Leaves & $18.3 \pm 1.7^{\mathrm{b}}$ & $20.0 \pm 2.0^{b}$ & $30.3 \pm 3.3^{a}$ & $30.4 \pm 3.2^{a}$ \\
\hline & & Roots & $13.6 \pm 1.4^{b}$ & $14.5 \pm 1.5^{\mathrm{a}}$ & $11.3 \pm 1.2^{b}$ & $17.7 \pm 1.8^{\mathrm{a}}$ \\
\hline & \multirow[t]{2}{*}{ As+13 mM TU } & Leaves & $8.7 \pm 0.9^{b}$ & $10.0 \pm 1.0^{b}$ & $14.8 \pm 1.8^{\mathrm{a}}$ & $10.4 \pm 1.1^{\mathrm{b}}$ \\
\hline & & Roots & $5.6 \pm 0.8^{c}$ & $6.8 \pm 0.7^{b}$ & $6.5 \pm 0.8^{b}$ & $10.5 \pm 1.1^{\mathrm{a}}$ \\
\hline \multirow{8}{*}{$\mathrm{H}_{2} \mathrm{O}_{2}$} & \multirow[t]{2}{*}{ Control } & Leaves & $4.3 \pm 0.4^{a}$ & $3.9 \pm 0.4^{b}$ & $3.6 \pm 0.4^{b}$ & $3.5 \pm 0.3^{b}$ \\
\hline & & Roots & $5.3 \pm 0.3^{a}$ & $5.4 \pm 0.5^{\mathrm{a}}$ & $4.9 \pm 0.5^{a}$ & $4.9 \pm 0.5^{a}$ \\
\hline & \multirow[t]{2}{*}{ As } & Leaves & $11.5 \pm 1.2^{\mathrm{a}}$ & $9.8 \pm 0.9^{a}$ & $9.6 \pm 0.9^{a}$ & $10.5 \pm 1.1^{a}$ \\
\hline & & Roots & $10.8 \pm 1.1^{\mathrm{a}}$ & $9.9 \pm 0.9^{a}$ & $9.8 \pm 0.9^{a}$ & $8.7 \pm 0.8^{\mathrm{a}}$ \\
\hline & \multirow[t]{2}{*}{ As+6.5 mM TU } & Leaves & $4.6 \pm 0.4^{a}$ & $4.1 \pm 0.4^{\mathrm{a}}$ & $4.3 \pm 0.4^{\mathrm{a}}$ & $3.3 \pm 0.3^{b}$ \\
\hline & & Roots & $4.9 \pm 0.5^{a}$ & $5.0 \pm 0.5^{\mathrm{a}}$ & $5.1 \pm 0.5^{\mathrm{a}}$ & $5.3 \pm 0.5^{a}$ \\
\hline & \multirow[t]{2}{*}{ As+13 mM TU } & Leaves & $2.4 \pm 0.2^{b}$ & $2.8 \pm 0.3^{a}$ & $3.0 \pm 0.3^{a}$ & $2.7 \pm 0.3^{a}$ \\
\hline & & Roots & $2.1 \pm 0.2^{b}$ & $3.3 \pm 0.3^{a}$ & $3.2 \pm 0.3^{a}$ & $3.8 \pm 0.4^{a}$ \\
\hline \multirow{8}{*}{ MDA } & \multirow{2}{*}{ Control } & Leaves & $4.6 \pm 0.4^{a}$ & $4.1 \pm 0.4^{a}$ & $3.7 \pm 0.3^{\mathrm{a}}$ & $3.9 \pm 0.4^{a}$ \\
\hline & & Roots & $4.9 \pm 0.5^{\mathrm{a}}$ & $4.9 \pm 0.5^{\mathrm{a}}$ & $4.8 \pm 0.5^{\mathrm{a}}$ & $5.1 \pm 0.5^{a}$ \\
\hline & \multirow[t]{2}{*}{ As } & Leaves & $16.5 \pm 1.6^{\mathrm{a}}$ & $10.7 \pm 1.1^{\mathrm{b}}$ & $10.5 \pm 1.1^{\mathrm{b}}$ & $10.8 \pm 1.1^{\mathrm{b}}$ \\
\hline & & Roots & $10.7 \pm 1.1^{\mathrm{a}}$ & $9.5 \pm 0.8^{a}$ & $9.4 \pm 0.9^{a}$ & $9.1 \pm 0.9^{a}$ \\
\hline & \multirow[t]{2}{*}{ As+6.5 mM TU } & Leaves & $4.2 \pm 0.5^{\mathrm{a}}$ & $3.9 \pm 0.4^{\mathrm{a}}$ & $3.9 \pm 0.4^{a}$ & $3.9 \pm 0.4^{a}$ \\
\hline & & Roots & $5.1 \pm 0.4^{a}$ & $5.1 \pm 0.4^{\mathrm{a}}$ & $5.3 \pm 0.5^{\mathrm{a}}$ & $5.4 \pm 0.5^{\mathrm{a}}$ \\
\hline & \multirow[t]{2}{*}{ As+13 mM TU } & Leaves & $3.3 \pm 0.3^{a}$ & $3.2 \pm 0.3^{a}$ & $3.1 \pm 0.3^{\mathrm{a}}$ & $3.6 \pm 0.4^{a}$ \\
\hline & & Roots & $3.2 \pm 0.3^{a}$ & $3.3 \pm 0.3^{a}$ & $3.5 \pm 0.4^{\mathrm{a}}$ & $3.0 \pm 0.2^{\mathrm{a}}$ \\
\hline \multirow[t]{8}{*}{ EL \% } & \multirow[t]{2}{*}{ Control } & Leaves & $3.8 \pm 0.4^{a}$ & $3.1 \pm 0.3^{b}$ & $3.7 \pm 0.4^{a}$ & $4.1 \pm 0.4^{a}$ \\
\hline & & Roots & $4.3 \pm 0.4^{a}$ & $4.3 \pm 0.4^{\mathrm{a}}$ & $4.6 \pm 0.5^{a}$ & $4.6 \pm 0.5^{a}$ \\
\hline & \multirow[t]{2}{*}{ As } & Leaves & $13.7 \pm 1.4^{\mathrm{a}}$ & $11.0 \pm 1.1^{\mathrm{a}}$ & $10.8 \pm 1.1^{\mathrm{a}}$ & $11.1 \pm 1.1^{\mathrm{a}}$ \\
\hline & & Roots & $16.9 \pm 1.7^{\mathrm{a}}$ & $12.6 \pm 1.2^{b}$ & $11.9 \pm 1.1^{\mathrm{b}}$ & $15.3 \pm 1.5^{a}$ \\
\hline & \multirow[t]{2}{*}{ As+6.5 mM TU } & Leaves & $4.2 \pm 0.4^{a}$ & $4.3 \pm 0.4^{a}$ & $3.9 \pm 0.4^{a}$ & $4.4 \pm 0.4^{a}$ \\
\hline & & Roots & $4.5 \pm 0.4^{a}$ & $4.0 \pm 0.4^{a}$ & $4.4 \pm 0.4^{a}$ & $4.6 \pm 0.5^{a}$ \\
\hline & \multirow[t]{2}{*}{$\mathrm{As}+13 \mathrm{mM}$ TU } & Leaves & $2.5 \pm 0.2^{a}$ & $2.9 \pm 0.3^{a}$ & $2.5 \pm 0.3^{a}$ & $2.6 \pm 0.3^{a}$ \\
\hline & & Roots & $2.8 \pm 0.3^{a}$ & $3.1 \pm 0.3^{\mathrm{a}}$ & $3.0 \pm 0.3^{a}$ & $2.8 \pm 0.3^{a}$ \\
\hline
\end{tabular}

Means \pm SE of four replicates treatment ${ }^{-1}$. Means followed by different lowercase letters in rows are significantly different at $P<0.05$ by ANOVA followed by Duncan's multiple range test. MG: Methylglyoxal, MDA: Malondialdehyde, EL: Electrolyte leakage, TU: Thiourea, As: Arsenic, SE: Standard error

dry mass accumulation, as suggested by significantly positive correlation between SDW and Chl A/B ratio. This has immense agronomic significance as photosynthetic pigments are one of the most vital internal physiological factors, which are believed to be the targets of As-induced toxicity in crop plants (Stoeva et al., 2005; Singh et al., 2007; Talukdar, 2013b).

As-induced significant increase in SOD activity and its reduction on imposition of As + TU treatment protocol suggested that As-treatment alone stimulated enhanced generation of superoxide radicals in leaves and roots of four genotypes. In contrast, TU application declined superoxide generation. During dismutation, SOD generates $\mathrm{H}_{2} \mathrm{O}_{2}$, which is another ROS across cellular membranes (Neill et al., 2002; Noctor et al., 2012). In this case, roles of three prominent AsA-GSH cycle enzymes against As-stress were studied. As-induced significant decline in APX activity severely jeopardized $\mathrm{H}_{2} \mathrm{O}_{2}$ scavenging in all four genotypes with more severe effects on L 9 and Pusa 4 than that on K 75 and PL 234. This resulted in overaccumulation of
$\mathrm{H}_{2} \mathrm{O}_{2}$ in both organs of As-treated crops. APX exclusively requires AsA as co-factor during ROS scavenging, the process which ultimately generates DHA (Asada, 2006). Low AsA pool in the As-treated plant organs particularly in roots might be due to reduced DHAR activity which regenerates AsA from DHA. Low DHAR activity also led to reduction in AsA redox which hovered around 0.5 in roots and 0.6 in leaves of the genotypes. The genotypic differences were evident from greater reduction of AsA and GSH level and their redox values in L 9 and Pusa 4 than those in K 75 and PL 234. Along with low AsA pool, decreased GR level failed to recycle enough GSSG to GSH. GR is a NADPH-dependent enzyme (Foyer and Noctor, 2011; Noctor et al., 2012), and failure to regenerate GSH resulted in low GSH redox and high GSSG level in photosynthetic and underground parts of all four genotypes with greater impacts on L 9 and Pusa 4 than K 75 and PL 234 genotypes. Thus, the three redox couples, i.e., AsA/DHA, GSH/GSSG, and NADP ${ }^{+} / \mathrm{NADPH}$ were badly affected with different magnitudes in four genotypes by As exposure alone. This accompanied by low GSTs and 


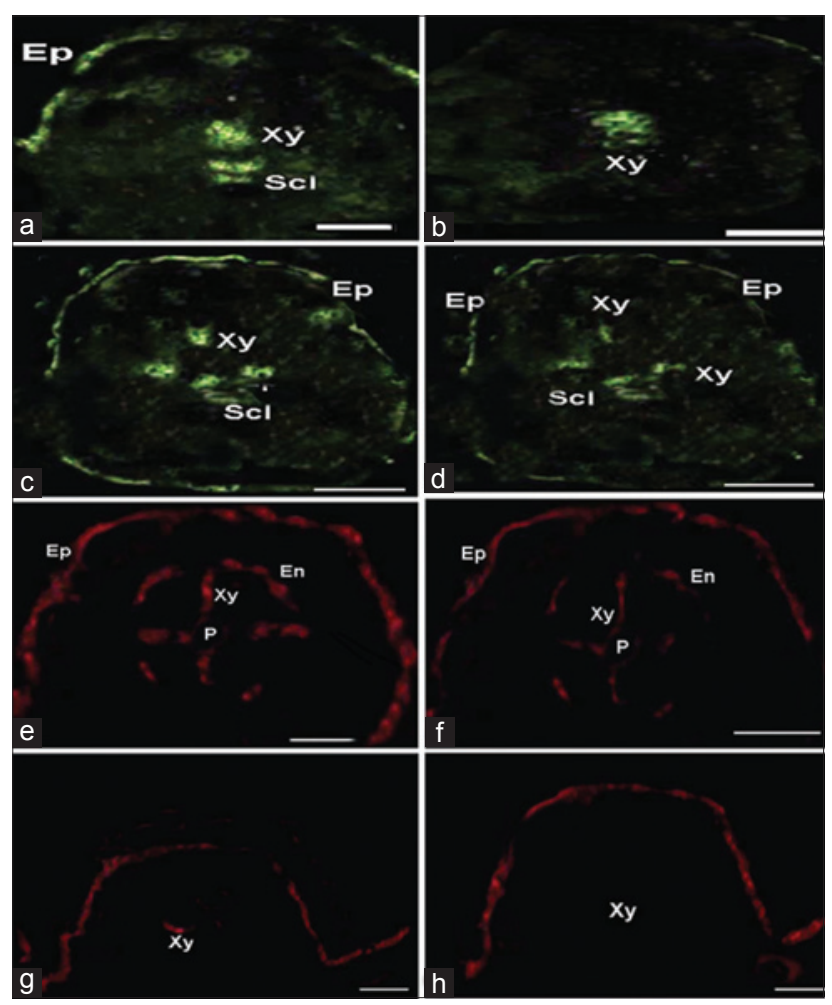

Figure 5: Representative photographs of reactive oxygen species imaging study by Confocal LASER Scanning Microscopy with dihydroethidium (DHE) (superoxide radicals-specific) and 2'7'-dichlorofluorescein diacetate $\left(\mathrm{H}_{2} \mathrm{O}_{2}\right.$-specific) dye showing mild green fluorescence in arsenic (As) $+6.5 \mathrm{mM}$ thiourea (TU) treated L 9 (a) leaf, (c) roots, As + 13 mM TU-treated L 9 (b) leaf, (d) roots, and mild DHE fluorescence in As + $6.5 \mathrm{mM}$ TU-treated $\mathrm{K} 75$ (e) roots, (g) leaf, and As $+13 \mathrm{mM}$ TU-treated K 75 (f) roots and (h) leaf sections. The results are representative of at least four independent experiments. Ep: Epidermis; En: Endodermis, Ms: Mesophyll cells; Scl: Sclerenchyma; Xy: Xylem; P-pith. Bars $=50 \mu \mathrm{m}$

CAT level resulted in accumulation of $\mathrm{H}_{2} \mathrm{O}_{2}$ and lipid peroxides, which consequently led to membrane damage and EL as the marks of As-induced oxidative stress in the four lentil genotypes.

The antioxidant defense component was further crippled due to As-induced reduction in capability of Gly I and II systems. As and cadmium-induced inhibition of antioxidant defense and Gly system was noticed in different other crops (Hossain et al., 2010; Hasanuzzaman et al., 2012). Reduced capability of Gly system may have resulted in overaccumulation of $\mathrm{MG}$ and crippled regeneration of GSH which may be another reason, besides low GR, for low GSH redox pool in present genotypes exposed to As-treatment only. Present result confirmed interaction between GSH-dependent antioxidant defense and Gly system in lentil genotypes under As stress. GSH/Gly plays pivotal roles in primary antioxidant defense, as previously monitored in salt-stressed crop plants (Yadav et al., 2005; Singla-Pareek et al., 2003; 2008; Hossain and Fujita, 2010). In this study, exogenously applied TU significantly ameliorated As-induced oxidative stress by preventing excess build-up of $\mathrm{H}_{2} \mathrm{O}_{2}$ and $\mathrm{MG}$, thereby protecting plants from As-induced oxidative damage. This was possible through modulation of primary antioxidant defense enzymes and Gly systems. In agreement with the present results, Gly system was found to be stimulated by exogenous GSH application in high temperature and drought stressed mung bean (Nahar et al., 2015a; 2015b), by external NO in As-stressed wheat (Hasanuzzaman and Fujita, 2013) and by exogenous proline and betaine in drought-stressed mung bean and lentil seedlings (Hossain et al., 2010; Molla et al., 2014). In this case, significant reduction in SOD activity and elevation of antioxidant as well as Gly I and II activities under As + TU treatment suggested TU-mediated modulation of entire antioxidant defense and MG detoxification system in fine tune to prevent ROS and MG overaccumulation. Interestingly, AsA and GSH levels as well as activities of AsA-GSH cycle enzymes and GSTs along with Gly I and II increased significantly in roots of both crops at As + TU treatment regimes when As accumulated at the much higher amount in their roots than the shoots. Obviously, harmonious elevation of antioxidant activity and MG-detoxification system was required together to sequester and detoxify As in roots. High DHAR and GR level maintained AsA and GSH redox around 0.8-0.9, favorable to maintain cellular reducing environment. High GSH pool also favored activity of GSTs to be stimulated to scavenge lipid peroxide and to sequester As in roots of four genotypes. Furthermore, stimulated Gly I and II system in presence of TU helped lentil genotypes to contain MG level comparable to control. Thus, alteration of entire antioxidant defense and Gly system, and their integrated responses clearly indicated that despite huge As accumulation, roots of all four genotypes were able to detoxify ROS and MG quite effectively. This consequently protected photosynthetic parts from As-induced oxidative damage by preventing upward translocation of As and maintained plant growth under As exposure. The results indicated TU-mediated upregulation of defense cascades against As-induced oxidative stress.

Occurrence of superoxide radicals and $\mathrm{H}_{2} \mathrm{O}_{2}$ was imaged by CLSM using the fluorescence probe DHE and DCF-DA, respectively. Occurrence of faint red (superoxide) and green $\left(\mathrm{H}_{2} \mathrm{O}_{2}\right)$ fluorescence in vascular bundle region indicated mild presence of free radicals in leaves and roots of untreated control. Contrastingly, bright fluorescence signals in vascular bundle regions, endodermis, sclerenchyma, mesophyll (leaf), and epidermis of As-treated leaves and roots of four genotypes 
suggested overaccumulation and abundant localization of superoxide and $\mathrm{H}_{2} \mathrm{O}_{2}$ in plant parts. Image analysis suggested significant decline of ROS distribution in presence of $6.5 \mathrm{mMTU}$ and further decline in presence of 13 mMTU. Significant taming of the fluorescence using TMP or AsA indicated specificity of DHE and DCF-DA for detection and imaging of superoxide radicals and $\mathrm{H}_{2} \mathrm{O}_{2}$, respectively, in crop tissues. Overproduction of superoxides and $\mathrm{H}_{2} \mathrm{O}_{2}$ due to toxic metals/metalloids was distinctly screened by CLSM study in pea, beans, alfalfa, and lupin roots (Ortega-Villasante et al., 2005; Rodr'iguez-Serrano et al., 2006; Talukdar and Talukdar, 2013). This study pointed out effective roles of TU as ROS scavenger, and although MG could not be detected by present preparation, it is suggested that use of TU considerably reduced the magnitude of ROS production in leaves and roots of four genotypes.

\section{CONCLUSION}

The present investigation revealed As-induced oxidative stress and consequent inhibition of growth traits in lentil genotypes. The four genotypes, however, differed in their responses under As and As + TU treatment protocols. The L 9 and Pusa 4 exhibited higher impact of As-treatment than K 75 and PL 234. However, exogenous application of TU significantly improved growth performances by modulating As-accumulation pattern, Gly system, and antioxidant defense mechanisms coordinately in leaves and roots of As-treated genotypes. ROS imaging study confirmed As-induced ROS generation and its significant amelioration by exogenous application of TU in both plant organs.

\section{ACKNOWLEDGMENT}

Author is grateful to Dr. Tulika Talukdar and students for their technical helps in biochemical study.

\section{REFERENCES}

Akladious SA. Influence of thiourea application on some physiological and molecular criteria of sunflower (Helianthus annuus L.) Plants under conditions of heat stress. Protoplasma 2014;251:625-38.

Asada K. Production and scavenging of reactive oxygen species in chloroplasts and their functions. Plant Physiol 2006;141:391-6.

Ashraf M, Harris PJ. Photosyntesis under stressful environments: An overview. Photosyntetica 2013;51:163-90.

Azizur Rahman M, Hasegawa H, Mahfuzur Rahman M, Nazrul Islam M, Majid Miah MA, Tasmen A. Effect of arsenic on photosynthesis, growth and yield of five widely cultivated rice (Oryza sativa L.) varieties in Bangladesh. Chemosphere 2007;67:1072-9.

Beyer WF Jr, Fridovich I. Assaying for superoxide dismutase activity: Some large consequences of minor changes in conditions. Anal Biochem 1987;161:559-66.

Bradford MM. A rapid and sensitive method for the quantitation of microgram quantities of protein utilizing the principle of protein-dye binding. Anal Biochem 1976;72:248-54.

Carlberg I, Mannervik B. Glutathione reductase. In: Alton M, editor. Methods in Enzymology. San Diego: Academic Press; 1985. p. 484-90.

Chaturvedi I. Effects of arsenic concentrations and forms on growth and arsenic uptake and accumulation by Indian mustard (Brassicsa juncea L.) genotypes. J Cent Eur Agric 2006; 7:31-40.

Dionisio-Sese M, Tobita S. Antioxidant responses of rice seedlings to salinity stress. Plant Sci 1998;135:1-9.

Finnegan PM, Chen W. Arsenic toxicity: The effects on plant metabolism. Front Physiol 2012;3:182.

Foyer CH, Noctor G. Ascorbate and glutathione: The heart of the redox hub. Plant Physiol 2011;155:2-18.

Foyer CH, Noctor G. Redox sensing and signaling associated with reactive oxygen in chloroplasts, peroxisomes and mitochondria. Physiol Plant 2003;119:355-64.

Griffith OW. Determination of glutathione and glutathione disulfide using glutathione reductase and 2-vinylpyridine. Anal Biochem 1980;106:207-12.

Gunes A, Pilbeam D, Inal A. Effect of arsenic-phosphorus interaction on arsenic-induced oxidative stress in chickpea plants. Plant Soil 2009;314:211-20.

Guo W, Hou YL, Wang SG, Zhu YG. Effect of silicate on the growth and arsenate uptake by rice (Oryza sativa L.) Seedlings in solution culture. Plant Soil 2005;272:173-81.

Gupta DK, Tripathi RD, Mishra S, Srivastava S, Dwivedi S, Rai UN, et al. Arsenic accumulation in root and shoot visa-vis its effects on growth and level of phytochelatins in seedlings of Cicer arietinum L. J Environ Biol 2008;29:281-6.

Hasanuzzaman M, Fujita M. Exogenous sodium nitroprusside alleviates arsenic-induced oxidative stress in wheat (Triticum aestivum L.) Seedlings by enhancing antioxidant defense and glyoxalase system. Ecotoxicology 2013;22:584-96.

Hasanuzzaman M, Hossain MA, Fujita M. Exogenous selenium pretreatment protects rapeseed seedlings from cadmiuminduced oxidative stress by upregulating antioxidant defense and methylglyoxal detoxification systems. Biol Trace Elem Res 2012;149:248-61.

Hodges DM, Delong JM, Forney CF, Prange RK. Improving the thiobarbituric acid reactive substances assay for estimating lipid peroxidation in plant tissues containing anthocyanin and other interfering compounds. Planta 1999;207:604-61 . 
Hossain MA, Fujita M. Evidence for a role of exogenous glycinebetaine and proline in antioxidant defense and methylglyoxal detoxification systems in mung bean seedlings under salt stress. Physiol Mol Biol Plants 2010;16:19-29.

Hossain MA, Hasanuzzaman M, Fujita M. Up-regulation of antioxidant and glyoxalase systems by exogenous glycinebetaine and proline in mung bean confer tolerance to cadmium stress. Physiol Mol Biol Plants 2010;16:259-72.

IIPR Vision, 2030. Printed \& Published by the Director, Indian Institute of Pulses Research (ICAR), Kanpur; 2011.

Law MY, Charles SA, Halliwell B. Glutathione and ascorbic acid in spinach (Spinacia oleracea) chloroplasts. The effect of hydrogen peroxide and of Paraquat. Biochem J 1983;210:899-903.

Li ZS, Zhen RG, Rea PA. 1-chloro-2,4-dinitrobenzene-elicited increase in vascular glutathione-S-conjugate transport activity. Plant Physiol 1995;109:177-85.

Lichtenthaler HK. Chlorophylls and carotenoids: Pigments of photosynthetic biomembranes. Methods Enzymol 1987;148:350-82.

Malik JA, Goel S, Kaur N, Sharma S, Singh S, Nayyar H. Selenium antagonises the toxic effects of arsenic on mung bean (Phaseolus aureus Roxb.) plants by restricting its uptake and enhancing the antioxidative and detoxification mechanisms. Environ Exp Bot 2012;77:242-8.

Molla MR, Ali MR, Hasanuzzaman M, Al-Mamun MH, Ahmed A, Nazim-Ud-Dowla MA, et al. Exogenous proline and betaine-induced upregulation of glutathione transferase and glyoxalase I in lentil (Lens culinaris) under drought stress. Not Bot Horti Agrobot 2014;42:73-80.

Nahar K, Hasanuzzaman M, Alam MM, Fujita M. Exogenous glutathione confers high temperature stress tolerance in mung bean (Vigna radiata L.) by modulating antioxidant defense and methylglyoxal detoxification system. Environ Exp Bot 2015a;112:44-54.

Nahar K, Hasanuzzaman M, Alam MM, Fujita M. Glutathioneinduced drought stress tolerance in mung bean: Coordinated roles of the antioxidant defence and methylglyoxal detoxification systems. AoB Plants 2015b;7:plv069.

Nakano Y, Asada K. Hydrogen peroxide is scavenged by ascorbate-specific peroxidase in spinach chloroplasts. Plant Cell Physiol 1981;22:867-80.

Neill S, Desikan R, Hancock J. Hydrogen peroxide signalling. Curr Opin Plant Biol 2002;5:388-95.

Noctor G, Mhamdi A, Chaouch S, Han Y, Neukermans J, Marquez-Garcia B, et al. Glutathione in plants: An integrated overview. Plant Cell Environ 2012;35:454-84.

Ortega-Villasante C, Rellán-Alvarez R, Del Campo FF, Carpena-Ruiz RO, Hernández LE. Cellular damage induced by cadmium and mercury in Medicago sativa. J Exp Bot 2005;56:2239-51.

Pandey M, Srivastava AK, D’Souza SF, Penna S. Thiourea, a ROS scavenger, regulates source-to-sink relationship to enhance crop yield and oil content in Brassica juncea (L.). PLoS One 2013;8:e73921.

Principato GB, Rosi G, Talesa V, Giovannini E, Uotila L. Purification and characterization of two forms of glyoxalase II from the liver and brain of Wistar rats. Biochim Biophys Acta 1987;911:349-55.

Rodr'1guez-Serrano M, Romero-Puertas MC, Zabalza A, Corpas FJ, Gómez M, Del Río LA, et al. Cadmium effect on oxidative metabolism of pea (Pisum sativum L.) roots. Imaging of reactive oxygen species and nitric oxide accumulation in vivo. Plant Cell Environ 2006;29:1532-44.

Sahu MP, Singh D. Role of thiourea in improving productivity of wheat (Triticum aestivum L.) J Plant Growth Regul 1995; 14:169-173.

Singh HP, Batish DR, Kohli RK, Arora K. Arsenic induced root growth inhibition in mung bean (Phaseolus aureus Roxb.) Is due to oxidative stress resulting from enhanced lipid peroxidation. Plant Growth Regul 2007;53:65-73.

Singla-Pareek SL, Reddy MK, Sopory SK. Genetic engineering of the glyoxalase pathway in tobacco leads to enhanced salinity tolerance. Proc Natl Acad Sci USA 2003;100:14672-7.

Singla-Pareek SL, Yadav SK, Pareek A, Reddy MK, Sopory SK. Enhancing salt tolerance in a crop plant by overexpression of glyoxalase II. Transgenic Res 2008;17:171-80.

Srivastava AK, Srivastava S, Penna S, D’Souza SF. Thiourea orchestrates regulation of redox state and antioxidant responses to reduce the $\mathrm{NaCl}$-induced oxidative damage in Indian mustard (Brassica juncea (L.) Czern.). Plant Physiol Biochem 2011;49:676-86.

Stoeva N, Berova M, Zlatev Z. Effect of arsenic on some physiological parameters in bean plants. Biol Plant 2005;9:293-6.

Talukdar D, TalukdarT. Coordinated response of sulfate transport, cysteine biosynthesis, and glutathione-mediated antioxidant defense in lentil (Lens culinaris Medik.) genotypes exposed to arsenic. Protoplasma 2014;251:839-55.

Talukdar D, TalukdarT. Superoxide-dismutase deficient mutants in common beans (Phaseolus vulgaris L.): Genetic control, differential expressions of isozymes, and sensitivity to arsenic. Biomed Res Int 2013;2013:782450.

Talukdar D. Arsenic-induced oxidative stress and its reversal by thiourea in mung bean (Vigna radiata (L.) Wilczek.) Genotype. Cent Euro J Exp Biol 2014;3:13-8.

Talukdar D. Arsenic-induced oxidative stress in the common bean legume, Phaseolus vulgaris L. Seedlings and its amelioration by exogenous nitric oxide. Physiol Mol Biol Plants 2013;19:69-79. 
Talukdar D. Glutathione deficiency in a grass pea (Lathyrus sativus L.) Mutant reveals major reshuffle in up-stream thiol cascade and downstream antioxidant defense under arsenate stress. Braz J Bot 2015;39:55-66.

Talukdar D. Plant growth and leaf antioxidant metabolism of four elite grass pea (Lathyrus sativus L.) Genotypes, differing in arsenic tolerance. Agric Res 2013b;2:330-9.

Tripathi RD, Tripathi P, Dwivedi S, Dubey S, Chatterjee S, Chakrabarty D, et al. Arsenomics: Omics of arsenic metabolism in plants. Front Physiol 2012;3:275.

Wang CQ, Chen M, Wang BS. Betacyanin accumulation in the leaves of C3 halophyte Suaeda salsa L. Is induced by watering roots with $\mathrm{H}_{2} \mathrm{O}_{2}$. Plant Sci 2007;172:1-7.

Wild R, Ooi L, Srikanth V, Münch G. A quick, convenient and economical method for the reliable determination of methylglyoxal in millimolar concentrations: The N-acetyl-L-cysteine assay. Anal Bioanal Chem 2012;403:2577-81.

Yadav SK, Singla-Pareek SL, Ray M, Reddy MK, Sopory SK. Methylglyoxal levels in plants under salinity stress are dependent on glyoxalase I and glutathione. Biochem Biophys Res Commun 2005;337:61-7. 